\title{
Coloduodenal Fistula in Right Colorectal Cancer: Case Report and Review of the Literature
}

\author{
Patricia Ciriano Hernández ${ }^{1^{*}} \mid$ Carlos Martínez Pinedo $\mid$ Rafael Picón Rodríguez $\mid$ Elisa \\ Jiménez Higuera | Daniel Sánchez Peláez | Ángel Fernández Camuñas | Jesús Martín
}

Fernández

\section{${ }^{1}$ General and Digestive System Surgery Hospital General Universitario de Ciudad Real Calle Obispo Rafael Torija 13005 Ciudad Real, Castilla la Mancha, Spain}

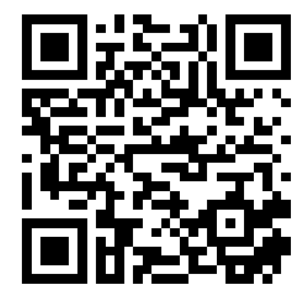

\section{1 | INTRODUCTION}

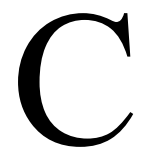
olorectal cancer is one of the most frequent malignancies among developed countries. Locally advanced cases range from 5 to $25 \%$. The specific invasion of the duodenum has been reported to account for $0,4 \%$. ((1), (2)

This clinical entity has become a rare event in present days. Colorectal cancer screening is widespread, and therefore early diagnosis and resection of the primary is the usual proceeding. That explains why isolated case reports are the most common source of current literature. ((3) , (4) , (5)

Symptoms of duodenal involvement of locally advanced right colorectal cancer depend on the primary tumor, the fistula or the metastatic burden. The most common are severe weight loss and malnutrition, metabolic disturbances, chronic blood loss and ane- mia, diarrhea or feculent vomiting. (1) (5)

Diagnosis is based on upper endoscopy and colonoscopy, which can also provide the biopsy for histopathological confirmation. The gold standard radiology test is the CT scan.

Management of malignant duodenal fistula poses a dilemma to surgeons. In some cases right colectomy

Supplementary information The online version of this article (https://doi.org/10.15520/jmrhs.v4i1.303) contains supplementary material, which is available to authorized users.

Corresponding Author: Patricia Ciriano Hernández

General and Digestive System Surgery Hospital General Universitario de Ciudad Real Calle Obispo Rafael Torija 13005 Ciudad Real, Castilla la Mancha, Spain Email: patricia.ciriano.hernandez@gmail.com 

THE LITERATURE

associated to duodenal resection or pancreaticduodenectomy is the only curative procedure, if confirmed absence of metastatic spread. This technically demanding procedure, becomes even more challenging due to the compromised patient's status. (1), (2)

\section{2 | MATERIALS AND METHODS}

A 75 year-old male presented with a three week history of feculent vomiting, abdominal pain and lassitude. Physical examination revealed weight loss and abdominal tenderness on right hypochondrium.

A contrast enhanced CT scan showed a locally advanced carcinoma of the right colon with an interface loss with the second part of the duodenum, suggestive of a coloduodenal fistula. (Fig 1,2).

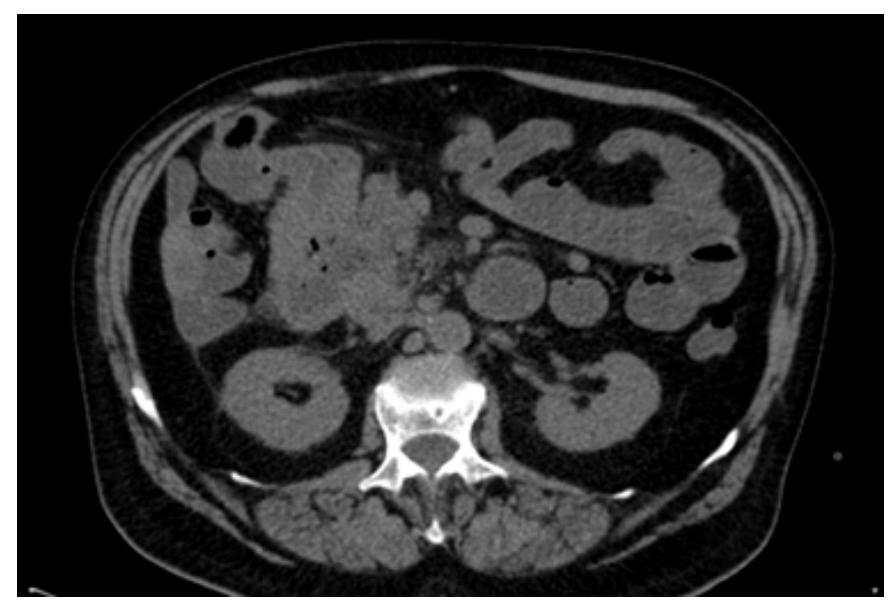

FIGURE 1: Coloduodenal fistula on CT scan

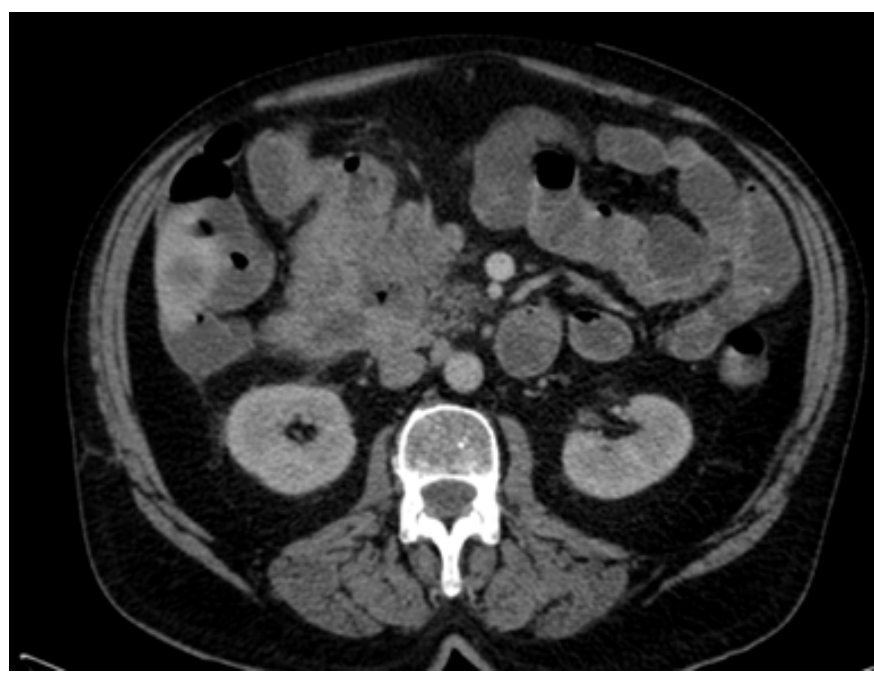

FIGURE 2: Coloduodenal fistula on enhanced CT scan
Bilobar liver metastasis and pancreatic infiltration were showed. (Fig 3, 4).

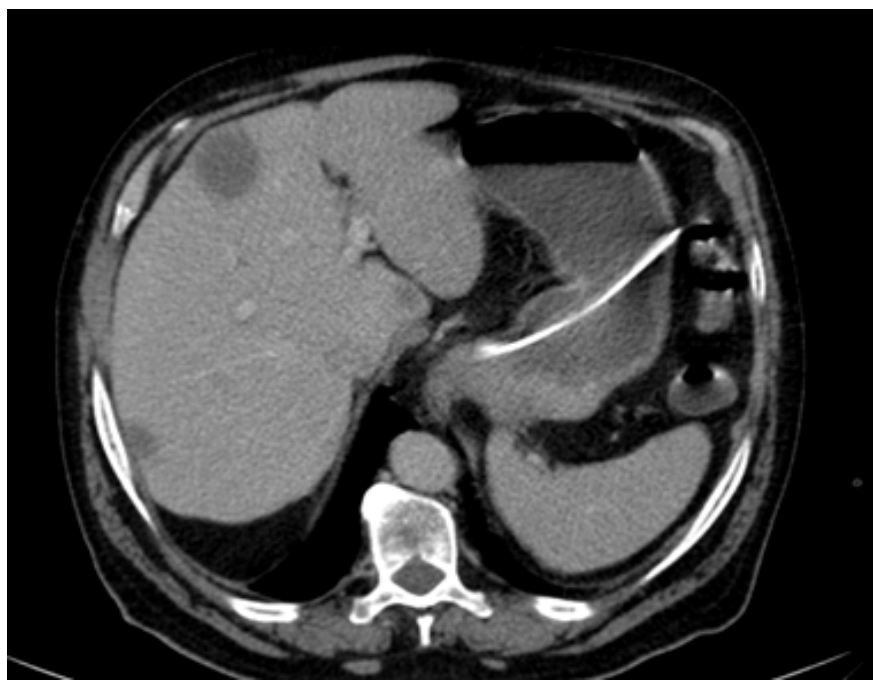

FIGURE 3: Bilobar liver metastasis on CT scan

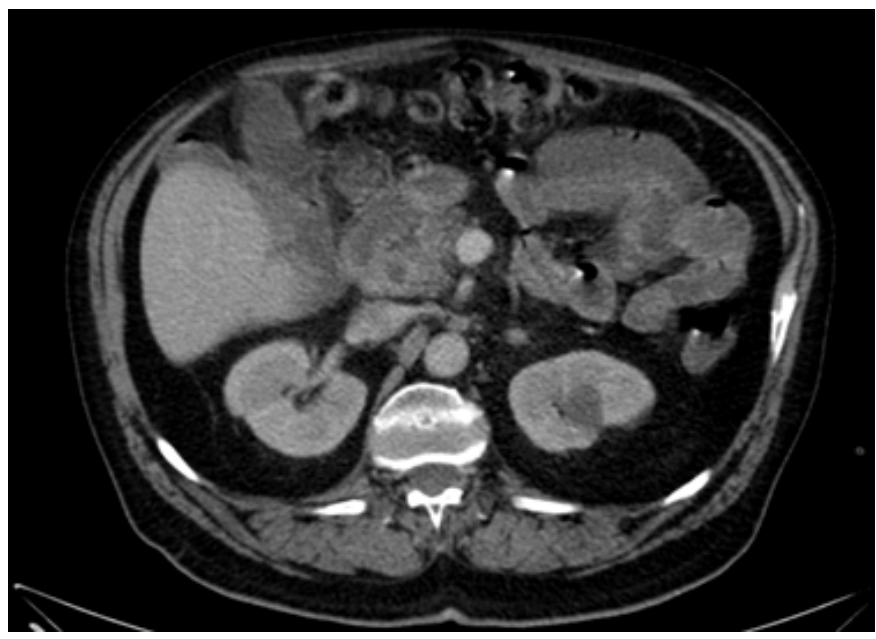

FIGURE 4: Pancreatic infiltration on CT scan

Colonoscopy confirmed an obstructive lesion previous to the hepatic flexure. In spite of it, histopathology showed a piece of normal colonic mucosa.

The patient's nutritional status and electrolyte disturbances were corrected prior to surgical treatment. Blood transfusion was also required.

The patient underwent exploratory laparotomy, finding an obstructive tumor of the hepatic flexure of the colon, extended to the second part of the duodenum. Presence of hepatic metastasis was confirmed as well as several peritoneal implants. Due to the poor prognosis and health status, palliative gastrojejunal 


\section{MEDICAL RESEARCH}

by pass and terminal ileostomy was performed.

Surgical histopathology confirmed moderately differentiated adenocarcinoma of the colon.

Postoperative course was complicated with periostomal cellulitis requiring drainage and exacerbation of chronic heart failure. The patient was discharged on postoperative day twenty-seventh.

Follow up was transferred to the palliative care unit. The patient died two months after hospital discharge.

\section{3 | DISCUSSION}

Colorectal cancer is one of the most common oncologic diagnosis in developed countries. (1), (2)

In spite of it, locally advanced colorectal cancer has become a less frequent diagnosis due to colorectal cancer screening, which is widespread in western countries. Early diagnosis leads to a prompt surgical approach and resection of the primary tumor. $(3,4)$

Vicinity organ invasion is more frequently diagnosed in cases of left colorectal cancer. Duodenal involvement and fistulization is a rare event, associated to right colorectal cancer, which often involves pancreas and also tends to show metastatic spread at diagnosis. As a consequence, the report of this clinical entity in literature sources is limited, mostly accounting for single cases. $(3,4,5)$

Recently Gupta et al. reported a retrospective series of nine patients diagnosed of coloduodenal fistula, most of them secondary to right colorectal cancer, and have proposed a classification based on the extent of invasion of the duodenal wall by the primary. This classification leads to different surgical techniques in order to manage the duodenal fistula: sleeve duodenal resection and primary closure, segmental duodenal resection and gastric or jejunal anastomosis to the remaining duodenum, and finally pancreaticduodenectomy. (1)

Fuks et al. also provided a series of fifteen cases of advanced right colon cancer involving the duodenal wall, performing almost the same surgical procedures even in cases of acute onset and need of emergency surgery. (2)

The high rate of severe complications associated to right colectomy if tumor is extended to duodenal wall, makes this surgical procedures unsuitable for many patients. Those complications range from medical issues as heart failure from postoperative complication as duodenal or pancreatic leak, intraabdominal abscess leading to septic shock, and eventually death. $(1,2,4)$

Authors agree on the need for pancreaticduodenectomy if absence of distant metastasis is confirmed, there is a real possibility of achieving tumor free margins and the patient status merits an aggressive and oncological procedure. $(1,2,4,5)$.

All cases reported were adenocarcinoma on histopathological examination. (1-5) Fuck et al. showed survival rates of $68 \%$ at one year and $53 \%$ at three years from resection, and recurrent rate of $46 \%$ in cases of T4 or lymph node involvement. (2)

In those cases presenting with metastasis or poor health status, curative procedures might not be an option. Most patients present severe malnutrition and weight loss at onset. Chronic blood loss and anemia are frequent symptoms. In addition, diarrhea, caused by colonic flora overgrowth contributes to worsen malnutrition and metabolic disturbances. In those cases palliative gastrojejunal by pass could be the only feasible option. Survival of those patients is less than a year, according to various publications. $(1,2$, 4)

\section{4 | CONCLUSION}

Malignant coloduodenal fistula is a rare event that happens under the diagnosis of right colon cancer. It has been usually associated to malnourished patients and a poor outcome and widespread metastatic disease. In spite of it, in selected cases surgery could be a feasible option, if complete removal of the primary and the fistula en bloc and negative margins are achieved.

\section{REFERENCES}

1. Gupta V, Kurdia KC, Kumar P, Yadav TD, Gulati A, Sinha SK, et al. Malignant colo-duodenal 
fistula: management based on proposed classification. Updates in Surgery. 2018;70(4):449458. Available from: https://dx.doi.org/10.1007/ s13304-018-0570-0. doi:10.1007/s13304-018-05 $70-0$.

2. Fuks D, Pessaux P, Tuech JJ, Mauvais F, Bréhant $\mathrm{O}$, Dumont $\mathrm{F}$, et al. Management of patients with carcinoma of the right colon invading the duodenum or pancreatic head. International Journal of Colorectal Disease. 2008;23(5):477481. Available from: https://dx.doi.org/10.1007/ s00384-007-0409-5. doi:10.1007/s00384-007-04 09-5.

3. Majeed TA, Gaurav A, Shilpa D, Preeti J, Sanjay S, Manisha S, et al. Malignant Coloduodenal Fistulas-Review of Literature and Case Report. Indian Journal of Surgical Oncology. 2011;2(3):205-209. Available from: https://dx. doi.org/10.1007/s13193-011-0099-x. doi:10.100 7/s13193-011-0099-x.

4. Soulsby R, Leung E, Williams N. Malignant coloduodenal fistula; case report and review of the literature. World Journal of Surgical Oncology. 2006;4:86-93.

5. Trikudanathan G, Farah K, Tiewala M. Malignant Coloduodenal Fistula: An Unusual Cause of Vomiting. ACG Case Reports Journal. 2015;2(1):137-138. Available from: https://dx. doi.org/10.14309/crj.2015.33. doi:10.14309/crj.2 015.33.

How to cite this article: P.C.H., C.M.P., R.P.R., E.J.H., D.S.P., A.F.C., J.M.F. Coloduodenal Fistula in Right Colorectal Cancer: Case Report and Review of the Literature. Journal of Medical Research and Health Sciences . 2020;1135-1138. https://doi.org/10.15520/jmrhs.v4i1.303 